\title{
STUDENT PROJECT Environmental influences on box blight epidemics
}

\author{
Lizzie Sharp', Clare Hurst², Jassy Drakulic ${ }^{3}$ \& Matthew Cromey ${ }^{4}$
}

\begin{abstract}
Calonectria pseudonaviculata and C. henricotiae are two recently differentiated fungal species responsible for box blight, a disease that threatens the Buxus genus. Infection can be introduced to gardens on new plants and is spread through the use of tools. The fungus survives on stem lesions and fallen leaves when spores are dispersed by rainsplash. In this study, 195 Calonectria UK isolates collected by the Royal Horticultural Society (RHS) Advisory Service were identified to species level. Detached stem assays were performed to assess how long stem and leaf lesions remain infectious, and their sensitivity to fungicides. A survey was also carried out at three National Trust properties on the effect of clipping on box blight distribution and severity. It was found that $C$. henricotiae was only present in and after 2011. C. henricotiae is more thermotolerant, and the increase in prevalence may be a result of increasing temperature and longer dry spells in the UK. Sporulation could occur multiple times on both stem and leaf lesions in humid conditions, although spore production dropped markedly after six sporulation events. Fungicides were effective at preventing spore production on stem lesions. Long dry spells may also reduce Calonectria's ability to sporulate, leading to limited box blight spread between plants.
\end{abstract}

\section{Introduction}

Plants in the genus Buxus (box) are popular ornamental plants and are of great economic value (Vitale et al., 2013). They are commonly clipped for aesthetic purposes (Gehesquière, 2014). The disease box blight was first recorded in the UK in 1994 by the Royal Horticultural Society (RHS) Gardening Advisory Service and in New Zealand in 1998. The symptoms of box blight are brown/ black lesions on the leaves and stems, and defoliation, both of which reduce the aesthetic value of clipped hedges. Severe infection can lead to the death of plants.

The origin of the pathogen is unknown, but it is now recorded in at least 21 countries. In 2002, the causal agent was described as Cylindrocladium pseudonaviculatum (Calonectria pseudonaviculata) (Crous et al., 2002) and a few months later as Cylindrocladium buxicola (Henricot \& Culham, 2002); these were later confirmed to be

\footnotetext{
${ }^{1}$ Lizzie Sharp was a Student Fellow at Royal Horticultural Society (RHS) for the duration of this study and a BSc student at the University of Cambridge.

Address: University of Cambridge, Department of Plant Sciences, Downing Street, Cambridge, CB2 3EA, UK.

${ }^{2}$ Clare Hurst was a Student Fellow at the RHS for the duration of this study and an MSci student at the University of East Anglia.

Address: University of East Anglia, School of Biological Sciences, Norwich Research Park, Norwich, NR4 7TJ, UK.

${ }^{3}$ Jassy Drakulic is a plant pathologist at the RHS, UK.

Address: RHS Garden Wisley, Woking, Surrey GU23 6QB.

${ }^{4}$ Matthew Cromey (corresponding author) is a plant pathologist at the RHS.

Address: RHS Garden Wisley, Woking, Surrey GU23 6QB, UK.

Email: matthewcromey@rhs.org.uk
} 
synonymous. Recent phylogenetic work (Gehesquière et al., 2014) has identified two phylogenetic clades which now form separate species: Calonectria pseudonaviculata (G1 clade, matching the original description) and C. henricotiae (G2 clade).

The earliest detection of Calonectria henricotiae was in 2005, where it caused a majority of 67.7 per cent of box blight isolates in Germany. In 2011, the first isolate of $C$. henricotiae was found in the UK, and the species accounted for 9.6 per cent of isolates. By 2012, a third of UK isolates were found to be $C$. henricotiae showing rapid growth, $C$. henricotiae, in comparison to C. pseudonaviculata, shows higher thermotolerance and a reduced sensitivity to fungicides (Gehesquière et al., 2016), meaning this species could be a greater threat. The RHS Gardening Advice Service receives diseased plant samples for diagnosis, including samples of box blight, which has provided the material for a large collection of Calonectria spp. isolates. The experiment described here uses a $\beta$-tubulin PCR-RFLP assay to categorise isolates from this collection as C. pseudonaviculata or C. henricotiae.

In damp conditions, a crop of white spores or conidia can be produced from stem and leaf lesions. These spores are dispersed by wind-driven rain (LaMondia \& Maurer, 2017). Conidia remain infective for three weeks in soil (Dart et al., 2015), however it is not known how long the spores remain viable on the leaves on which they are produced, how climatic conditions affect their longevity, or whether lesions develop more spores once the first ones have been washed off. This project investigates the longevity of spores on leaves in different conditions and the number of times leaf and stem lesions can repeatedly sporulate.

Less is known about the biology of stem lesions than that of leaf lesions. This project seeks to determine how long the fungus remained alive within stem lesions, and to see whether fungicides are better used to prevent the formation of stem lesions or kill the fungus within stem lesions once they have been formed.

Movement of infected plants through trade is the primary way in which box blight spreads between countries (Healy, 2014); formally planted gardens containing imported plants may have a greater presence of Calonectria than in wild Buxus landscapes. We also hypothesise that clipping Buxus plants directly impacts the distribution and development of box blight due to changes in the plant architecture. Clipped plants have little airflow and densely branching formations which would promote splash dispersal on a single plant and increase humidity to promote infection. Furthermore, tools have been described as a way Calonectria can be transported from infected to healthy plants (Vitale et al., 2013). In this study, samples will be taken from Buxus plants in formally clipped, lightly clipped and untrained forms from the National Trust properties Box Hill, Clandon Park and Hatchlands Park. The effect of plant morphology on box blight disease severity and distribution will be assessed.

In this project, we aim to characterise the species of Calonectria on Buxus in the UK and possible changes in population structure over time. We also aim to determine the factors that influence spore production and viability as well as to identify possible influences of plant architecture on box blight epidemics.

\section{Materials and methods}

\section{Fungal isolates}

The RHS Wisley Calonectria collection comprises 195 isolates, collected from 2001 to 2019. Samples of box blight were received from RHS Advisory enquiries or collected by 
RHS staff. Conidia from sporulating lesions were plated onto Potato Dextrose Agar (PDA) and Calonectria isolates transferred into pure culture. Isolates stored on PDA slopes were plated on PDA plates and incubated in the dark at $20^{\circ} \mathrm{C}$ for five to seven days until sufficient mycelium was available for DNA extraction.

New isolates were collected from a survey of Buxus which was conducted to investigate the effect of clipping on disease severity (described below). Cuttings from box plants showing symptoms of box blight (black leaf lesions, black stem lesions, areas of dieback) were taken from nine areas at Hatchlands Park, four areas at Box Hill and five areas at Clandon Park (Surrey, UK). Cuttings were placed in damp chambers and were left for three to five days to sporulate. Spores were then plated on PDA and were grown as above.

\section{PCR-based discrimination of isolates}

Mycelium was scraped off each plate and the DNeasy ${ }^{\circledR}$ PowerSoil ${ }^{\circledR}$ Kit was used to extract DNA. Following this, species identification was achieved by PCR of the $\beta$-tubulin gene followed by restriction digestion (Gehesquière et al., 2016). PCR reactions ( $20 \mu \mathrm{l})$ were prepared containing $1 \mu$ LNA template, 7 $\mu \mathrm{l}$ distilled water, $10 \mu$ Biomix Red Master Mix, $1 \mu$ l Forward Primer T1 and $1 \mu$ l Reverse Primer T2. The thermocycling programme consisted of a 5-minute initial preheating at $94^{\circ} \mathrm{C}$, followed by 35 cycles of denaturation at $94^{\circ} \mathrm{C}$ for 30 seconds; annealing at $55^{\circ} \mathrm{C}$ for 45 seconds; and extension at $72^{\circ} \mathrm{C}$ for 45 seconds, before a final extension step at $72^{\circ} \mathrm{C}$ for 10 minutes. From this, $18 \mu$ l distilled water, $2 \mu \mathrm{l}$ 10X Buffer $O$ and $2 \mu \mathrm{l}$ FastDigest Notl restriction enzyme (Fermentase) were added to $10 \mu$ l of each PCR product and incubated for 16 hours at $37^{\circ} \mathrm{C}$. Digested products were loaded onto 1.5 per cent agarose gel for electrophoresis at $120 \mathrm{~V}$ for 30 minutes and visualised on a UV visualiser.

\section{Obtaining spores for detached stem inoculation}

Spores for the detached stem inoculations were obtained by flooding and scraping sporulating cultures of isolate 17/02 of Calonectria henricotiae (grown on PDA) three times. The resulting spore solution was vortexed and filtered through a layer of cheesecloth. The concentration of the spore solution was determined using a haemocytometer, diluted with sterile distilled water (SDW) to 20,000 spores $\mathrm{ml}^{-1}$ for all experiments except fungicide sensitivity assays which used 14,000 spores $\mathrm{ml}^{-1}$.

\section{Inoculations}

Box plants and cuttings taken from hedges of common box at Deers farm (Wisley, Surrey) were inoculated by running gloved fingers dipped in spore solution up each stem/the back of the leaves (finger inoculation), sometimes accompanied by spraying spore solution evenly onto the foliage using a spray bottle (spray inoculation). This was followed by two days in a dew chamber to encourage infection. Leaves with lesions were stored at $4{ }^{\circ} \mathrm{C}$ until needed.

\section{Spore production on infected leaves}

To produce spores, leaves from inoculated cuttings were arranged abaxial side up in petri dishes on top of filter paper (Whatman No. 1) soaked with $1 \mathrm{ml}$ of SDW. Leaves were incubated for one or two days, and were examined under a dissecting microscope for sporulation daily.

\section{Longevity of spores in wet or dry conditions}

Leaves were arranged on petri dishes on either wet or dry filter paper to simulate wet 
or dry weather conditions. Four leaves from each condition (one from each replicate plate) were selected at random twice each week and submerged individually in $3 \mathrm{ml}$ water. The container with the leaf in water was vortexed to dislodge the spores. Spore solution was spread using an inoculation loop over a circle of water agar with a diameter of approximately $2 \mathrm{~cm}$. The plate was then left overnight. The sections of agar over which spore solution had been spread were cut out of the plate, stained with a drop of methyl blue to stain the conidia, and put under the $10 \times$ lens of a compound microscope. The numbers of germinated and non-germinated spores in four different fields of view were counted, and the overall percentage of germinated spores calculated. The criterion for a spore to be considered germinated was that its germ tube should be greater than its length. Only spores fully in the field of view were counted.

\section{Repeated sporulation on leaf lesions}

Seven sporulating leaves were submerged separately in $3 \mathrm{ml}$ water and vortexed to wash off the spores. The concentration of the spore solution was measured using a haemocytometer, and the leaves were removed from the solution, dried with tissue, and replaced onto the damp filter paper to see if they would sporulate again. After five days, they were examined again under the dissecting microscope. If they had re-sporulated they were washed and dried again and the concentration of the spore solution was measured again. The process was repeated every time they re-sporulated (every three to five days). The areas of the abaxial sides of each of each leaf were estimated by tracing round the leaves on graph paper and counting the number of small squares within this outline. This allowed the density of spores on the leaf to be calculated to control for the effect of leaf size on number of spores produced.

Role of stem lesions in pathogen survival, disease spread and epidemiology

Stem lesions were generated by finger inoculation of cuttings of common box from Deers farm using spore solution at 20,000 spores $/ \mathrm{ml}$. Newly inoculated cuttings were incubated in a dew chamber for two days to prevent the spores from drying out and so help the fungus to infect the leaves. They were then left in water in a room with temperature-controlled cycles $\left(20^{\circ} \mathrm{C}\right.$ day and $18{ }^{\circ} \mathrm{C}$ night) at $<50$ per cent humidity. Approximately 12 stem lesions were taken from these finger-inoculated cuttings each week for five weeks and incubated in petri dishes to assess sporulation.

The first twelve stem lesions to be left to sporulate were washed once every three or four days after sporulating for the first time to see how many times they would produce a new crop of spores. Lesions could be classified as either 'sporulating' or 'not' after examination using a dissecting microscope.

\section{Efficacy of fungicides against stem lesions}

Sixteen Buxus sempervirens (common box) plants in pots were arranged outdoors in a $4 \times 4$ grid with a gap of about $3 \mathrm{~cm}$ between plants. The plants were randomly allocated to one of four treatments: uninoculated control, fungicide prior to inoculation, fungicide post inoculation and no fungicide. A systemic fungicide, a mixture of $0.125 \mathrm{~g} / \mathrm{l}$ of trifloxystrobin and $0.125 \mathrm{~g} / \mathrm{l}$ of tebucanazol commercially available as Fungus Fighter Plus (Bayer Garden, UK), was used for the 
fungicide treatments. Inoculation was carried out on 16 July 2018 using a spore solution at 14,000 spores/ml. Uninoculated control plants were mock inoculated with water. Fungicide was applied to the 'prior to inoculation' group three days before inoculation, and to the 'post inoculation' group three weeks after inoculation (once the plants' symptoms were well developed).

Plants were blind assessed for symptoms twice a week. Numbers of stem lesions and leaf lesions were counted. All stem lesions were collected and plated as described above four weeks after inoculation (one week after the second application of fungicide) to assess sporulation and determine whether the fungus was active in the stem lesions after treatment with fungicide.

\section{Distribution of box blight}

A survey was carried out in July 2019 at three National Trust properties in Surrey: Hatchlands Park (Fig. 1), a site containing natural, semi-clipped and clipped Buxus plants; Box Hill (Fig. 2), a site with only natural Buxus plants; and Clandon Park, a site with semi-clipped and clipped Buxus plants. Samples were taken from 15 sites in Hatchlands Park (3 clipped, 3 semi-clipped and 9 natural), 4 sites in Box Hill and 5 sites in Clandon Park (2 clipped, 3 semi-clipped). Cuttings were taken where dieback or black leaf and stem lesions were found, and disease symptoms and severity were recorded.

Cuttings were placed in damp chambers and left for three to five days to sporulate. The presence of box blight was confirmed in

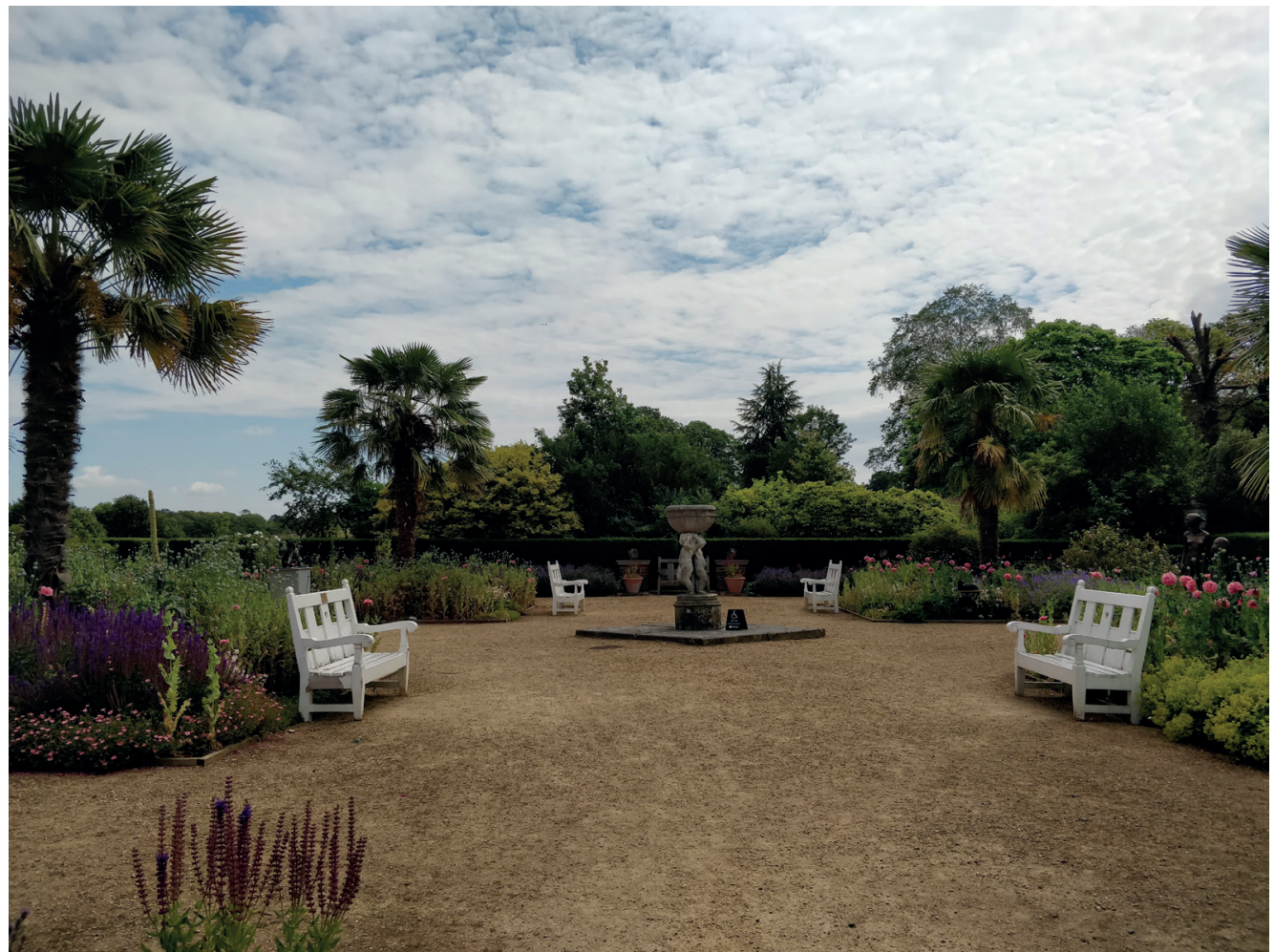

Fig. 1 Site of a former Buxus parterre at Hatchlands Park (National Trust) that was removed due to a severe outbreak of box blight. The surrounding tall Buxus hedge remains and has small areas of damage from previous box blight outbreaks. Photo: RHS/Jassy Drakulic. 


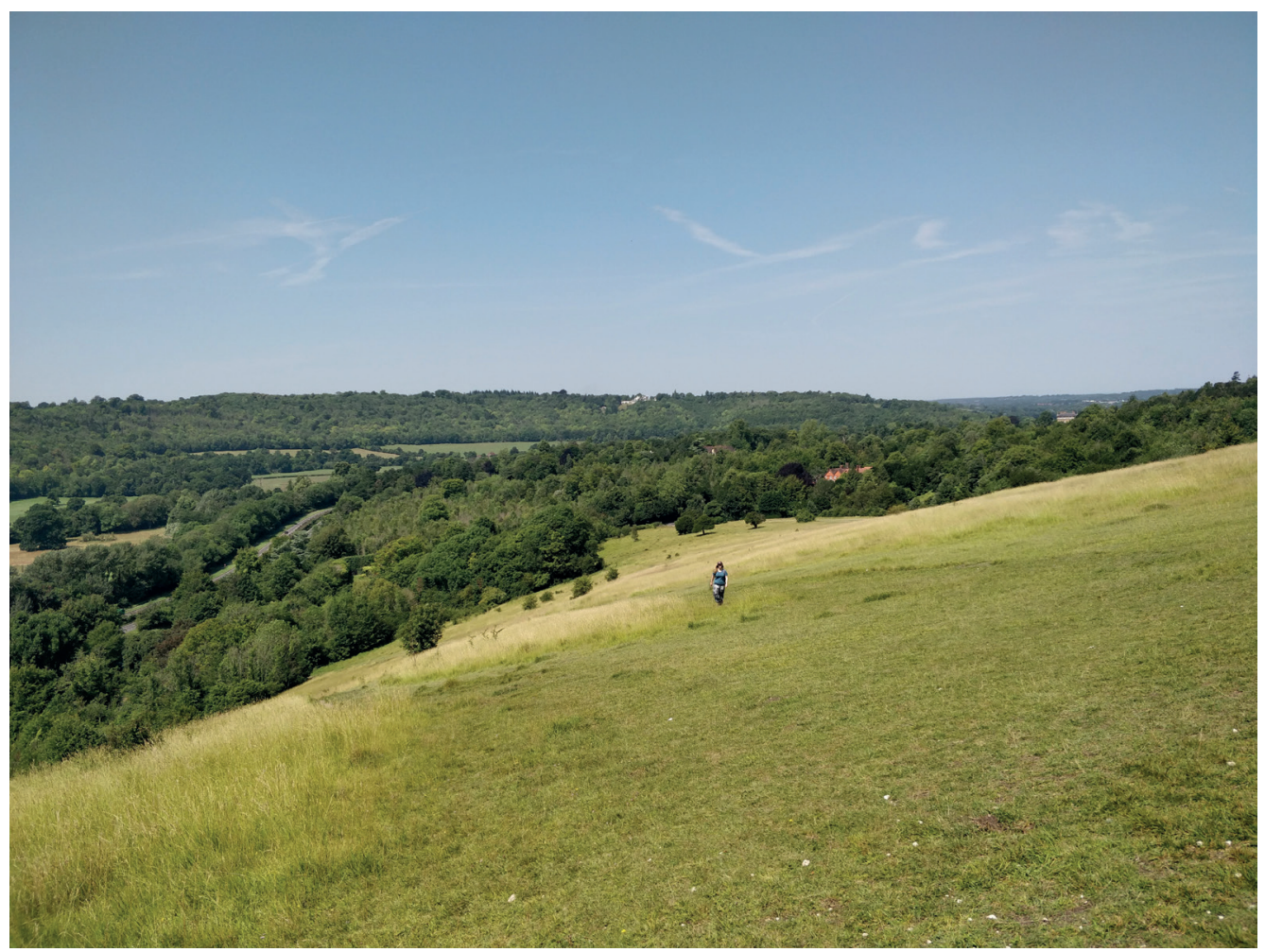

Fig. 2 Box Hill, Surrey, showing native Buxus. Photo: RHS/Jassy Drakulic.

samples where Calonectria spp. spores could be identified by light microscopy.

\section{Statistical methods}

To determine longevity of lesion infectivity, the effect of the number of days since sporulation in either wet or dry conditions (independent variables) on the percentage of germinating spores (dependent variable) in a lesion was analysed using analysis of variance (ANOVA), including the significance of the interaction between the effects in wet and dry conditions. The effect of the number of times a leaf had sporulated (independent variable) on the number of spores produced per $\mathrm{mm}^{2}$ (dependent variable) over time also used ANOVA, considering the interaction between the number of days left to sporulate and the number of times it sporulated. Likewise, the effects of fungicide treatment and the length of time since inoculation on the number of stem lesions produced were analysed using ANOVA, testing for interactions between the time since inoculation and fungicide treatment. Normality was assessed by inspection of residual plots. Analysis of fungicide treatment on the percentage of stem lesions that sporulated was performed by t-test. All analyses were performed in $\mathrm{R}$ ( $\mathrm{R}$ Core Team, 2017).

\section{Results}

\section{Species distribution}

Of 195 UK Calonectria isolates collected from the RHS Members Advisory Service, 188 were C. pseudonavaculata and 7 were C. henricotiae. Prior to 2011, all isolates were C. pseudonavaculata $(n=169)$. The first isolate to be collected of $C$. henricotiae was 
in 2011. In that year 25 per cent of isolates were C. henricotiae. In 2012, 23.08 per cent of isolates were $C$. henricotiae. No isolates were collected between 2013 and 2016, and only a small number were collected in 2017 to 2019. Of these, 40 per cent were C. henricotiae (Table 1). Only one isolation was successful from the survey of National Trust properties. The isolate was collected from the formally clipped parterre at Clandon Park.

\section{Longevity of spores on leaf lesions}

There was an initial increase in the viability of spores produced on leaves (as measured by the percentage of spores able to germinate) in both damp and dry conditions (Fig. 3). The percentage of spores germinating from leaves kept in dry conditions rose from 15 per cent one day after sporulating to about 80 per cent five days after sporulating. After this point, viability of spores in damp conditions remained high ( $\geq 65$ per cent) while there was a steady decline in viability of spores in dry conditions, to an average of 15 per cent 31 days after sporulation (Fig. 4). Using data from day three onwards (to avoid attempting to model the initial increase and subsequent decrease with the same linear model), a

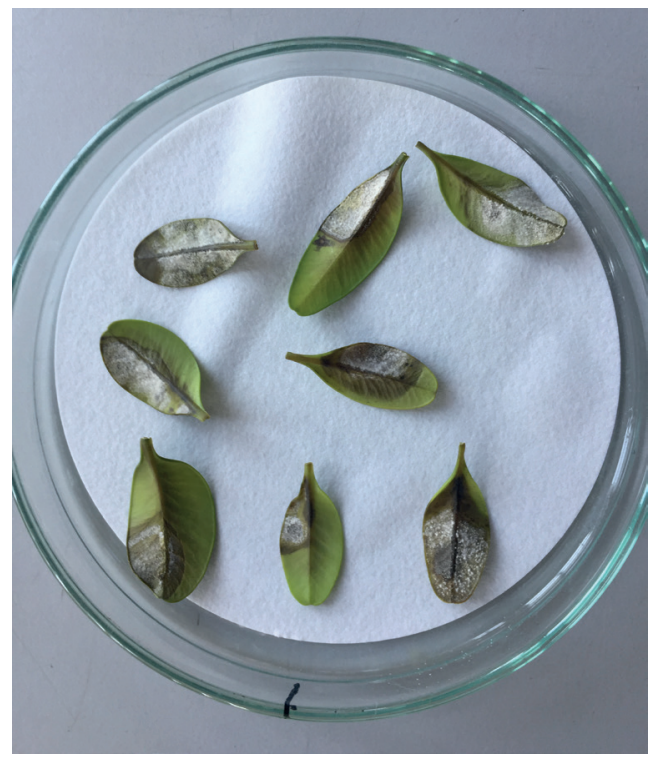

Fig. 3 Calonectria henricotiae spores produced on the undersides of Buxus leaves incubated in high humidity. Photo: RHS/Lizzie Sharp.

significant interaction between conditions and time since sporulation on percentage germinated was found $\left(F_{1,67}=46.6, p<0.001\right.$, $\eta^{2}=0.100$ ). There was considerable variation in spore viability between spores on leaves kept in the same conditions for the same length of time.

Some leaves sporulated at least nine times. There was a wide range in the

\begin{tabular}{|c|c|c|}
\hline Year & C. pseudonaviculata & C. henricotiae \\
\hline pre 2007 & 130 & 0 \\
\hline 2007 & 31 & 0 \\
\hline 2008 & 8 & 2 \\
\hline 2011 & 6 & 3 \\
\hline 2012 & 10 & 1 \\
\hline 2017 & 1 & 0 \\
\hline 2018 & 1 & 1 \\
\hline 2019 & 1 & \\
\hline
\end{tabular}

Table 1 Numbers of isolates of Calonectria species collected in different years 


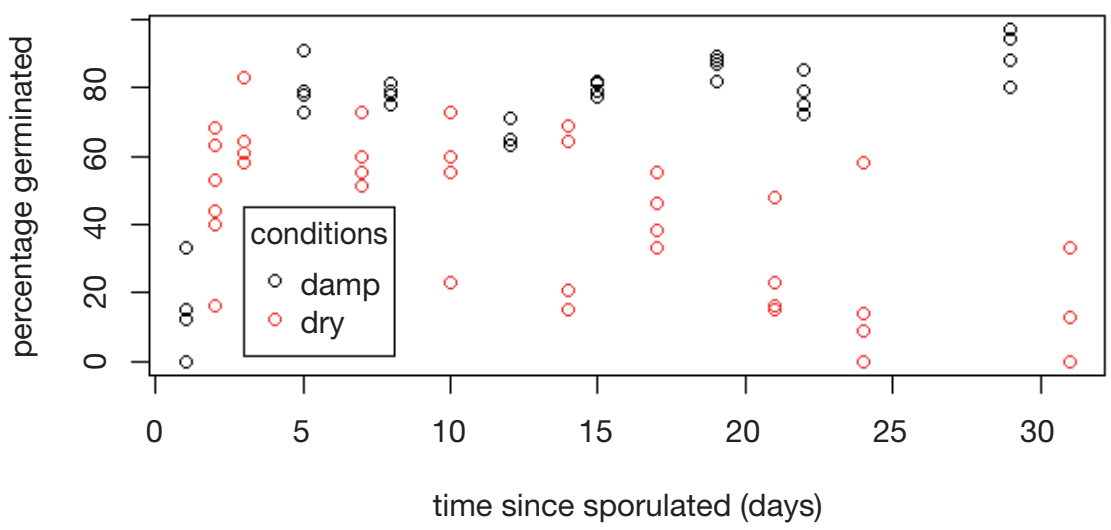

Fig. 4 Percentage of spores germinating from leaves maintained in damp or dry conditions for 1 to 32 days.

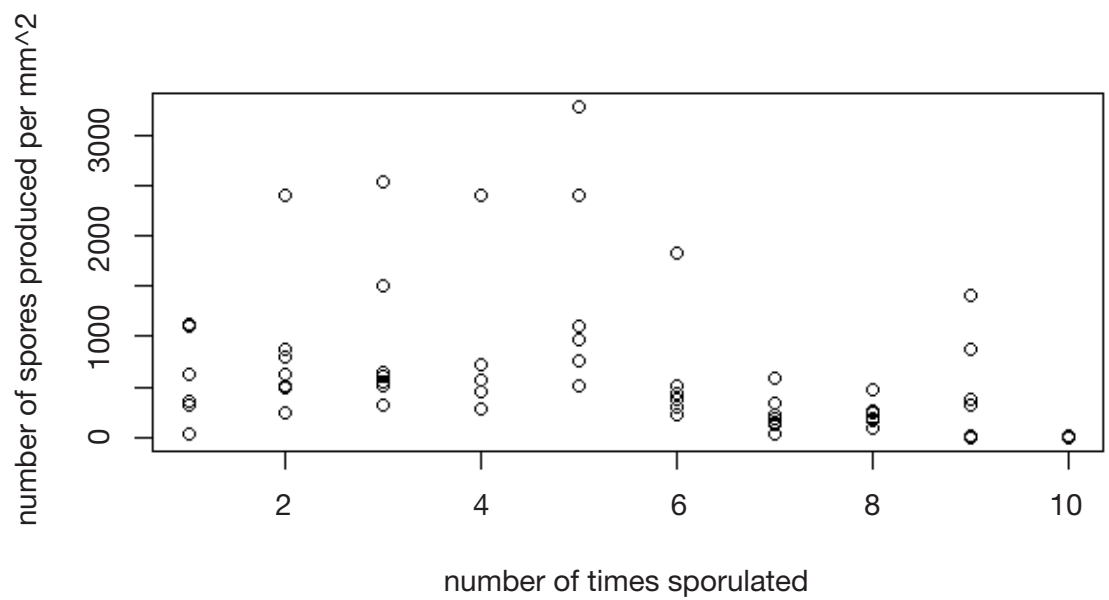

Fig. 5 Spore production on leaves incubated one to ten times in high humidity.

density of spores that were produced on a re-sporulating leaf at each sporulation event (Fig. 5), the largest range observed being between 506 spores $/ \mathrm{mm}^{2}$ and 3,285 spores/ $\mathrm{mm}^{2}$, both produced on leaves re-sporulating for the fourth time. ANOVA analysis shows a relationship between the number of times a leaf sporulated and the number of spores it produced per sporulation event $\left(F_{1,68}=9.7\right.$, $p=0.0027, \eta^{2}=0.0593$ ). The location of spore production also changed over time. Initially, leaves produced a carpet of spores spread over the lower leaf surface. When re-sporulating, this carpet (where present) appeared less densely packed with spores. Spores were most often produced in clusters towards the central leaf vein and petiole. These clusters occasionally formed on the upper leaf surface in addition to the lower leaf surface.

\section{Stem lesions: role in pathogen survival, disease spread and epidemiology}

Stem lesions sporulated when placed in a petri dish on filter paper soaked in $1 \mathrm{ml}$ of water (Fig. 6). As stem lesions aged, the percentage that sporulated fell from the initial value of 100 per cent, but it did not 


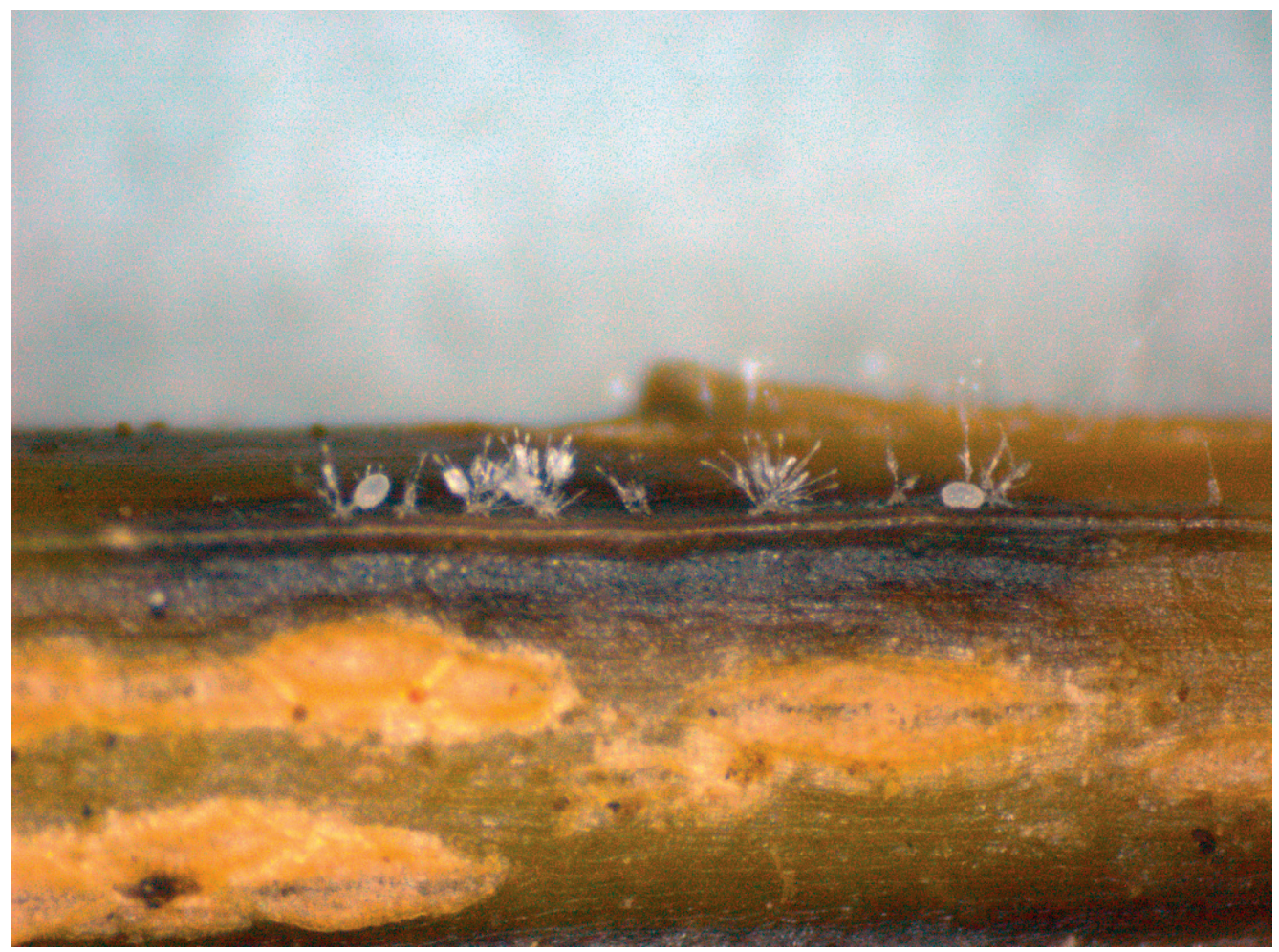

Fig. 6 Calonectria pseudonaviculata sporulating on box blight lesion on a Buxus stem. Photo: RHS/Matthew Cromey.

drop below 85 per cent over the five-week course of the experiment (Table 2).

Stem lesions sporulated at least ten times following removal of spores and re-incubation (Fig. 7). Not all stem lesions re-sporulated the first time the stems were washed, but by the time the stem lesions had been washed four times, all the stem lesions re-sporulated. Some stem lesions produced no further spores after seven sporulation events. The distribution of spores on the stems changed over the course of sporulation events, from being limited to the lesions themselves to covering much of the stem adjacent to the lesion as well.

\section{Efficacy of fungicides against stem lesions}

The systemic fungicide was completely effective against the formation of stem lesions

\begin{tabular}{|c|c|}
\hline $\begin{array}{c}\text { Time since } \\
\text { inoculated (weeks) }\end{array}$ & $\begin{array}{c}\text { \% of stem lesions } \\
\text { sporulating }\end{array}$ \\
\hline 1 & 100 \\
\hline 2 & 86 \\
\hline 3 & 88 \\
\hline 4 & 86 \\
\hline 5 & 89 \\
\hline
\end{tabular}

Table 2 Percentage of stem lesions sporulating from samples of approximately 12 stem lesions taken weekly from cuttings inoculated with spore solution at 20,000 spores $/ \mathrm{ml}$ and left in a petri dish on filter paper soaked in $1 \mathrm{ml}$ of water.

when used as a preventative treatment (Fig. 8). ANOVA showed a significant interaction effect between time since inoculation and treatment on the number of stem lesions $\left(F_{120,3}=18.1, p<0.05\right)$. Post hoc testing using Tukey's HSD (honestly significant difference) 


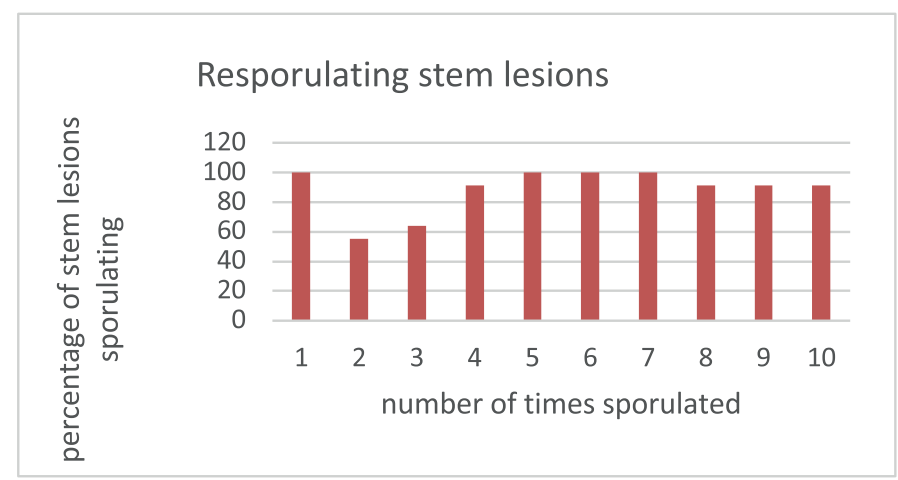

Fig. 7 Percentage of lesions sporulating on stems incubated one to ten times in high humidity.

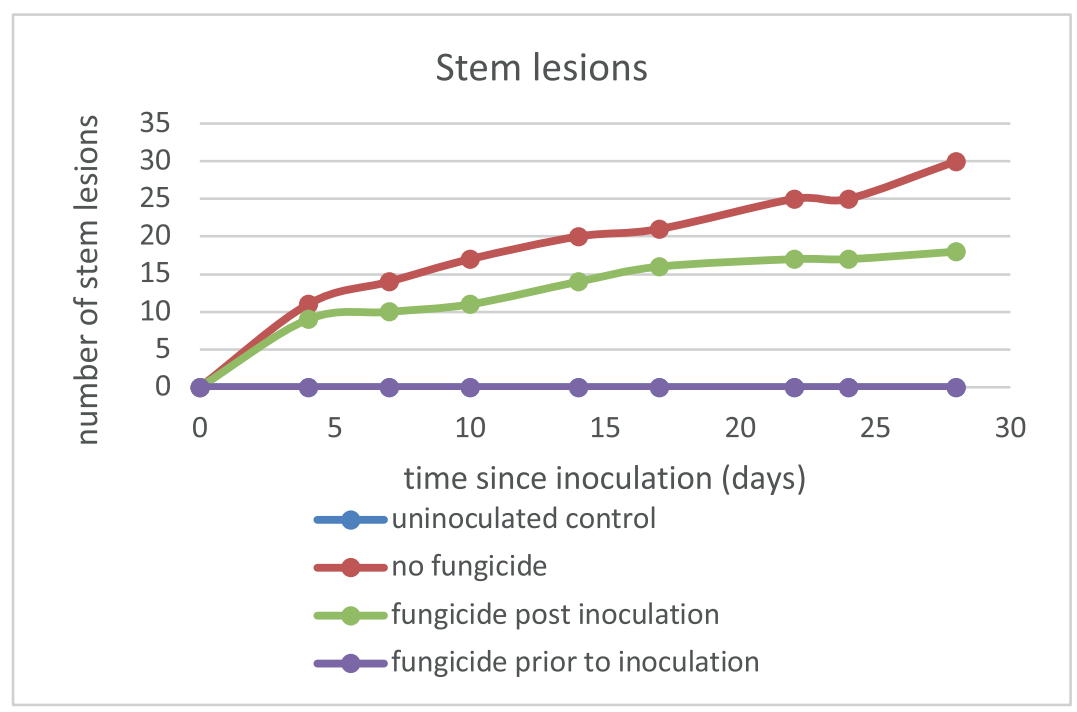

Fig. 8 Number of lesions developing over time on inoculated plants treated with fungicide prior to inoculation, after inoculation, or left untreated.

test showed significant differences $(p<0.05)$ between all treatment groups except the uninoculated controls and the group treated with fungicide prior to inoculation. The plants treated with fungicide prior to inoculation developed a few leaf lesions, although symptom development was slower than in untreated plants and the disease symptoms were not as severe (Fig. 9).

None of the stem lesions taken from plants treated with fungicide after the development of symptoms sporulated when incubated. An average of $21.5 \%$ of stem lesions from plants not treated with fungicide sporulated. The difference between the mean percentage sporulating with and without fungicide was not significant at the level of $P<0.05$ $\left(\mathrm{t}_{3(0.05)}=-2.69, P=0.075\right)$.

\section{Survey of box blight distribution in gardens and natural settings The survey at National Trust properties detected box blight at Hatchlands Park and}




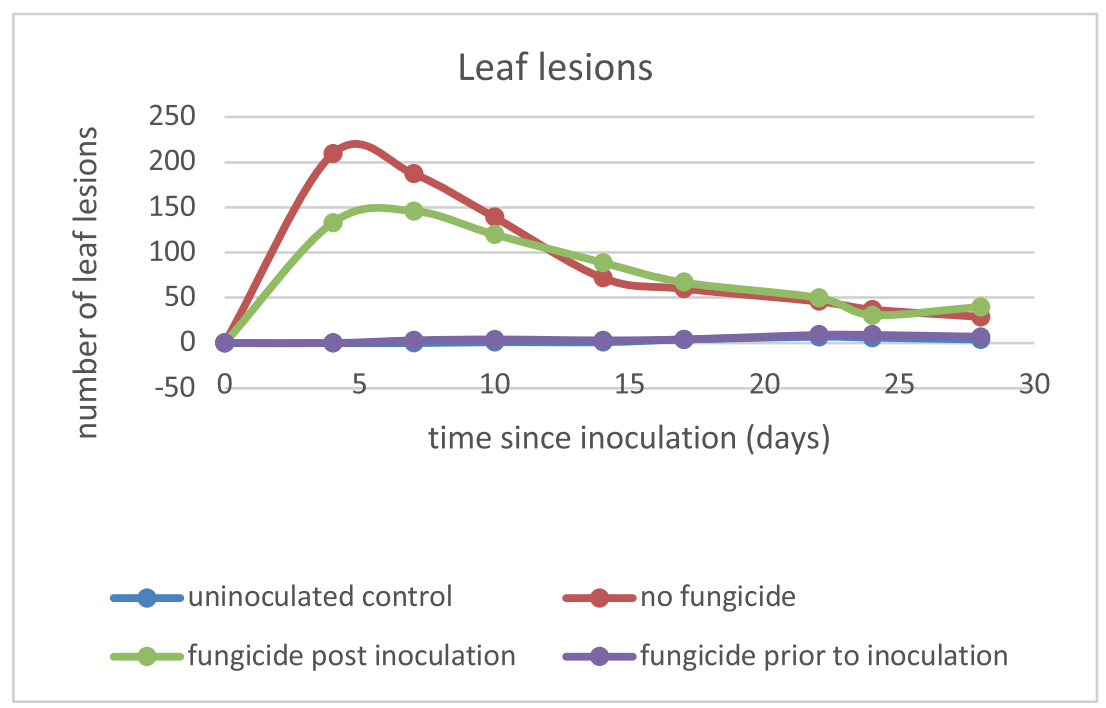

Fig. 9 Numbers of leaf lesions on plants over time on inoculated plants treated with fungicide prior to inoculation, after inoculation, or left untreated. Reductions in numbers of lesions are due to defoliation following infection.

Clandon Park (Table 3). Hatchlands Park showed areas of previous epidemic among the clipped hedges (Fig. 10), but no new lesions indicating infection occurred in previous years. One active blight infection was found among the natural Buxus plants (Fig. 11), and none among the semi-clipped planting area. Clandon Park showed signs of a previous epidemic on the formally clipped parterre (Fig. 12), but none in the formally clipped Dutch Garden. Two tiers of hedging within the parterre showed considerable areas of previous defoliation with old stem lesions still visible. The taller hedging had

\begin{tabular}{|l|c|c|c|c|c|c|}
\hline Location & Characteristic & $\begin{array}{c}\text { Number } \\
\text { of sample } \\
\text { sites }\end{array}$ & $\begin{array}{c}\text { Leaf } \\
\text { lesions }\end{array}$ & $\begin{array}{c}\text { Stem } \\
\text { lesions }\end{array}$ & $\begin{array}{c}\text { Lesion } \\
\text { age }\end{array}$ & $\begin{array}{c}\text { Presence of } \\
\text { box blight } \\
\text { sporulation }\end{array}$ \\
\hline Hatchlands & Natural & 8 & None & None & N/A & None \\
\hline Hatchlands & Natural & 1 & Present & Present & New & Present \\
\hline Hatchlands & Semi-clipped & 3 & None & None & N/A & None \\
\hline Hatchlands & Clipped & 3 & Present & Present & Old & None \\
\hline Box Hill & Natural & 4 & None & None & N/A & None \\
\hline Clandon & Semi-clipped & 1 & Present & Present & New & Present \\
\hline Clandon & Semi-clipped & 1 & Present & Present & Old & None \\
\hline Clandon & Semi-clipped & 1 & None & None & N/A & None \\
\hline Clandon & Clipped & 2 & Present & Present & Old & None \\
\hline
\end{tabular}

Table 3 Characteristic and number of sample sites in Hatchlands Park, Box Hill and Clandon Park. Presence of leaf and stem lesions, age of lesions and presence of box blight in samples taken. 


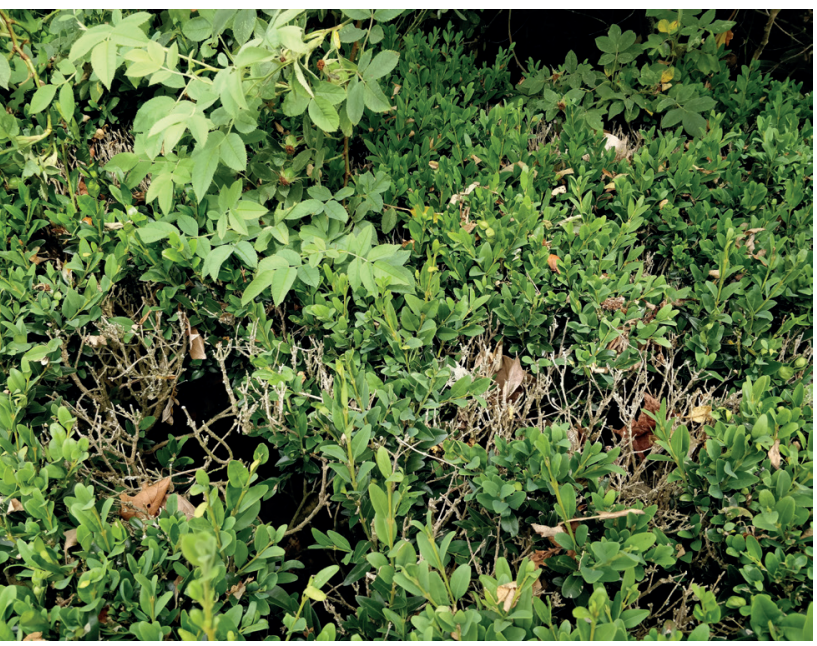

Fig. 10 Formally clipped box hedge showing damage from a previous box blight outbreak. Photo: RHS/Jassy Drakulic. put on considerable regrowth and the new stems had active lesions present, from which a successful isolation was made.

\section{Discussion}

In this study, we have characterised UK populations of Calonectria on Buxus and identified environmental and plant architectural factors that influence spore production and survival as well as box blight disease development.

\section{Calonectria henricotiae was present}

in the collection only after 2011, which supports the previous results indicating that this species first arrived in the UK in that year (Gehesquière et al., 2016). The isolate recovered from Clandon Park was

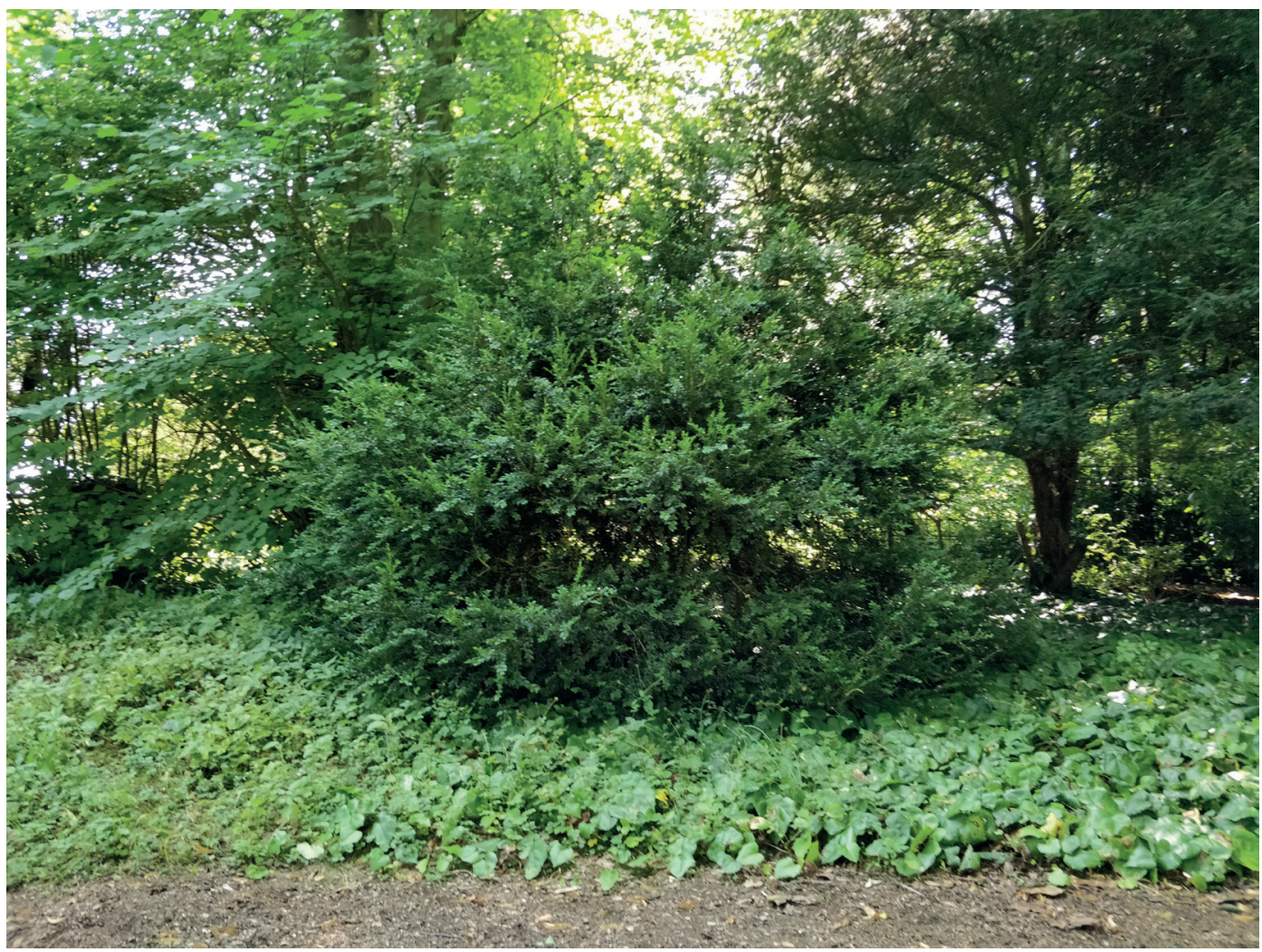

Fig. 11 Unclipped Buxus at Hatchlands Park (National Trust). A small area of box blight is present at the base of the plant. Photo: RHS/Jassy Drakulic. 


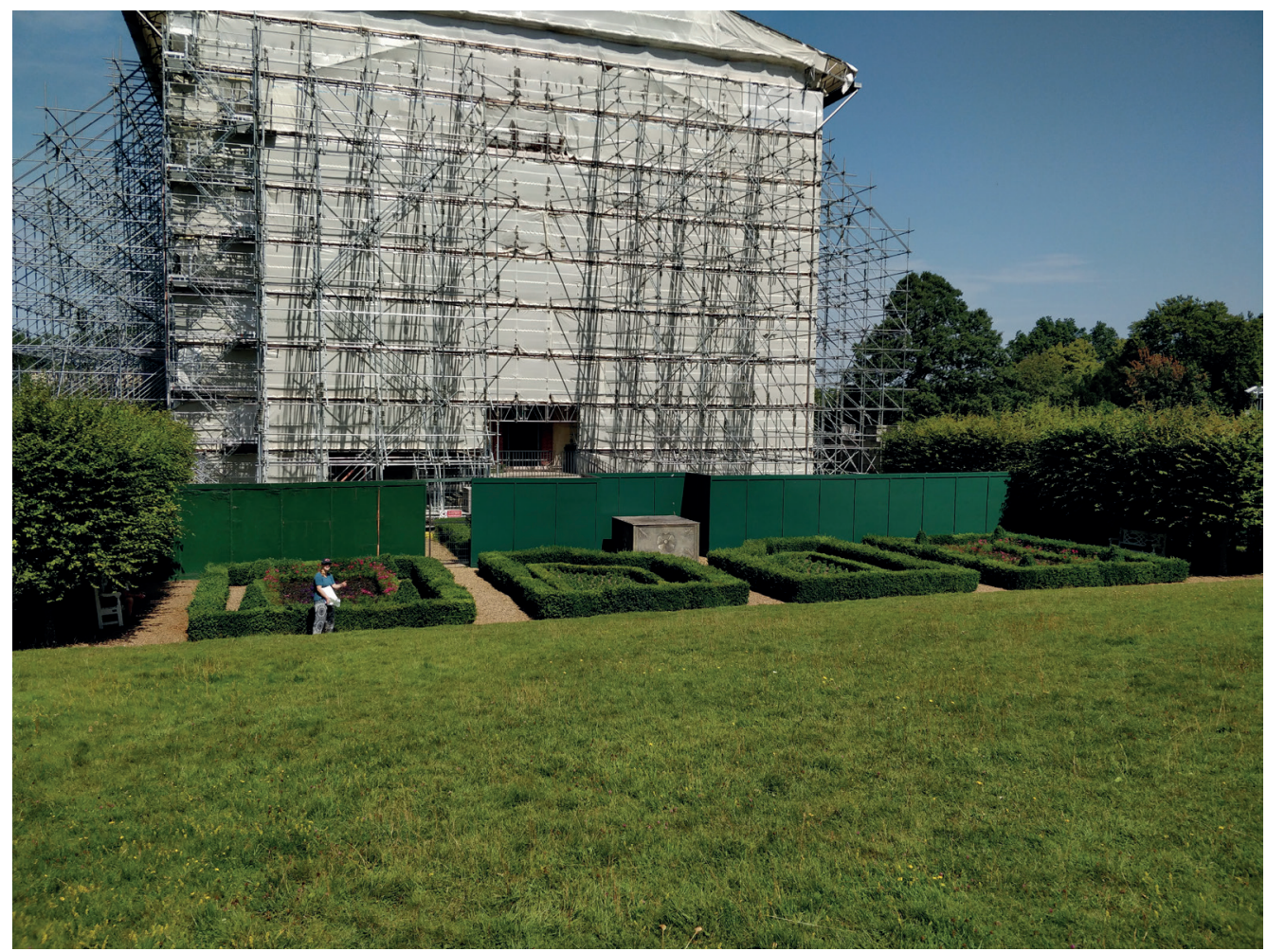

Fig. 12 Formally clipped parterre at Clandon Park (National Trust). Active box blight was present in the taller outer hedges, while the inner hedges showed damage from a previous outbreak. Photo: RHS/Jassy Drakulic.

from an established infection focus and was of $C$. pseudonaviculata. The hedges of the parterre have been planted for over 100 years (Historic England, 2003), so the potential to introduce $C$. henricotiae from new plants is low. As such, this identification, and as C. henricotiae isolates from 2018 and 2019 were those sent to the RHS Advisory Service from member gardens, supports the hypothesis that $C$. henricotiae is a recent introduction to the UK. A larger survey of UK garden isolates would be needed to determine whether $C$. henricotiae has largely replaced earlier populations of C. pseudonaviculata.

Spores of Cylindrocladium buxicola developed on box blight leaf and stem lesions on Buxus sempervirens in humid conditions. The results of germination tests show that spore viability is low when spores are first produced on leaves. This may be because it takes some time - up to five days - to become fully mature or that they may produce a factor that prevents the spores from germinating when they are initially produced. This would help to ensure that spores did not germinate on the leaf, but only once the spores had been dispersed.

Spores are dispersed from leaves by wind-driven rain. The results of the tests for longevity of spores on leaves show that spore viability falls in dry conditions. This suggests that infected leaves will become less of a risk to healthy plants in long periods without rain to disperse the spores because they have fewer viable spores. In wet weather, however, the viability of spores will remain high, and if the spores splash off in the rain more can be 
produced within a few days. High humidity is also required for infection once spores reach a new plant.

The changes in the distribution of spores to include areas outside the original lesions on leaves and stems as they re-sporulate multiple times suggests that the fungus grows within the plant tissue in search of new resources. Re-sporulation of leaf and stem lesions is, however, eventually limited by resources available within the plant tissues. This is demonstrated by the reduction in spore density over time.

When used as a preventative measure, the fungicide was completely effective in preventing the formation of stem lesions (although plants did develop a few leaf lesions). It was also fully effective at preventing sporulation of the fungus on stem lesions when used as a curative treatment.

The box blight survey showed that the most severe box blight infections had occurred on formally clipped areas, although box blight lesions were also found on occasion in natural and semi-clipped areas. The pathogen only sporulated on samples from natural and semi-clipped plants, whereas infections in formally clipped areas appeared to be inactive. Although box blight had clearly developed in these areas, it is unlikely that there had been re-infection in the last year. These findings are consistent with the hypothesis that pruning practices can influence disease severity, although a larger sample size and structured sampling would improve the confidence in these conclusions. In the parterre of Clandon House, shorter, more tightly pruned hedges showed damage from previous box blight epidemic.

An increasing number of dry spells in the UK over the past few years may be a cause of this apparent decline in box blight prevalence (Webster et al., 2017), as leaf wetness is required for Calonectria to infect Buxus (LeBlanc et al., 2018), as well as for the pathogen to sporulate on lesions (Shishkoff et al., 2016). The increase in temperatures in the UK (Webster et al., 2017), along with the sensitivity of $C$. pseudonaviculata to temperatures over $30^{\circ} \mathrm{C}$ (Shishkoff et al., 2016), may have caused this species to diminish. C. henricotiae has increased thermotolerance (Miller et al., 2018), thus an increase in the proportion of isolates identified as $C$. henricotiae over time would reflect the increase in UK temperature (Webster et al., 2017). The thermotolerance of $C$. henricotiae may provide it with a selective advantage, which could increase the likelihood of its spread to countries in southern Europe (Gehesquière et al., 2016). It may also result in greater damage in a changing UK climate, with poorer fungicide control possible due to its lack of fungicide sensitivity. Our results show that environment plays a major role in survival and spread of the box blight pathogen and that there is the potential to better manage box blight by modifying the microclimate surrounding plants.

\section{Acknowledgements}

We thank RHS members for provision of sample material for Calonectria isolations. Most of the early isolates were collected by Beatrice Henricot. We thank the National Trust for access to their properties and especially Piers Horry. Thanks also to the British Society for Plant Pathology and the British Mycological Society for funding the two summer studentships which formed the basis of this research. The RHS is a registered charity, no. $222879 /$ SCO38262. 


\section{References}

CROUS, P.W., GROENEWALD, J.Z. \& HILL, C.F. (2002). Cylindrocladium pseudonaviculatum sp. nov. from New Zealand, and new Cylindrocladium records from Vietnam. Sydowia, 54: 23-34.

DART, N., HONG, C., CRAIG, C.A., FRY, J.T. \& HU, X. (2015). Soil inoculum production, survival and infectivity of the boxwood blight pathogen Calonectria pseudonaviculata. Plant Disease, 99: 1689-1694.

GEHESQUIÈRE, B. (2014). Cylindrocladium buxicola nom. cons. prop. (syn. Calonectria pseudonaviculata) on Buxus: molecular characterization, epidemiology, host resistance and fungicide control. PhD dissertation, Ghent University. Available online: https://biblio.ugent.be/publication/4377930/ file/4377949 (accessed September 2019).

GEHESQUIÈRE, B., CROUCH, J.A., MARRA, R.E., VAN POUCKE, K., RYS, F., MAES, M., GOBIN, B., HOFTE, M. \& HEUNGENS, K. (2016). Characterization and taxonomic reassessment of the box blight pathogen Calonectria pseudonaviculata, introducing Calonectria henricotiae sp. nov. Plant Pathology, 65: 37-52.

HEALY, S. (2014). Biology and management of box blight caused by Cylindrocladium buxicola. MSc dissertation, Guelph University. Available online: https://pdfs.semanticscholar.org/b6dd/3c2ef16eba f23a4886ceacd30c3fec5fa97f.pdf (accessed August 2019).

HENRICOT, B. \& CULHAM, A. (2002).

Cylindrocladium buxicola, a new species affecting Buxus spp., and its phylogenetic status. Mycologia, 94: 980-997.

HISTORIC ENGLAND (2003). Clandon Park. Available online: https://historicengland.org.uk/ listing/the-list/list-entry/1001171\#contributionsbanner (accessed August 2019).

LAMONDIA, J.A. (2015). Management of Calonectria pseudonaviculata in boxwood with fungicides and less susceptible host species and varieties. Plant Disease, 99: 363-369.

LAMONDIA, J.A. \& MAURER, K. (2017).

Calonectria pseudonaviculata dispersal mechanisms and implications for boxwood blight management. Phytopathology, 107(12): 201-202.

LEBLANC, N., SALGADO-SALAZAR, C. \& CROUCH, J. (2018). Boxwood blight: an ongoing threat to ornamental and native boxwood. Applied Microbiology and Biotechnology, 102: 4371-4380.

MILLER, M., SHISHKOFF, N. \& CUBETA, M. (2018). Thermal sensitivity of Calonectria henricotiae and Calonectria pseudonaviculata conidia and microsclerotia. Mycologia, 110(3): 546-558.

R CORE TEAM (2017). R: A language and environment for statistical computing. $R$ Foundation for Statistical Computing, Vienna. Available online: https://www.R-project.org (accessed August 2018).

SHISHKOFF, N., \& CAMP, M. (2016). The effect of different temperatures and moisture levels on survival of Calonectria pseudonaviculata in boxwood leaves and twigs and as microsclerotia produced in culture. Plant Disease, 100(10): 2018-2024.

VITALE, A., CROUS, P., LOMBARD, L. \& POLIZZI, G. (2013). Calonectria diseases on ornamental plants in Europe and the Mediterranean basin: an overview. Journal of Plant Pathology, 95(3): 463-476.

WEBSTER, E., CAMERON R. \& CULHAM, A. (2017). Gardening in a Changing Climate. Royal Horticultural Society, London. 